\title{
Aberrant crypt foci and colon cancer: comparison between a short- and medium-term bioassay for colon carcinogenesis using dimethylhydrazine in Wistar rats
}

M.A.M. Rodrigues,

L.A.G. Silva,

D.M.F. Salvadori,

J.L.V. de Camargo and

M.R. Montenegro
Departamento de Patologia, Faculdade de Medicina, Universidade Estadual Paulista, Botucatu, SP, Brasil

\section{Correspondence \\ M.A.M. Rodrigues \\ Departamento de Patologia, Box 564 \\ Faculdade de Medicina, UNESP \\ 18618-000 Botucatu, SP \\ Brasil \\ Fax: + 55-14-6821-2348 \\ E-mail: mariar@fmb.unesp.br}

Research supported by FAPESP (No. 98/0706-9).

Received September 25, 2001 Accepted January 2, 2002

\section{Abstract}

Aberrant crypt foci (ACF) in the colon of carcinogen-treated rodents are considered to be the earliest hallmark of colon carcinogenesis. In the present study the relationship between a short-term (4 weeks) and medium-term (30 weeks) assay was assessed in a model of colon carcinogenesis induced by dimethylhydrazine $(\mathrm{DMH})$ in the rat. Sixweek-old male Wistar rats were given subcutaneous injections of DMH $(40 \mathrm{mg} / \mathrm{kg})$ twice a week for 2 weeks and killed at the end of the 4 th or 30th week. ACF were scored for number, distribution pattern along the colon and crypt multiplicity in $0.1 \%$ methylene-blue wholemount preparations. ACF were distinguished from normal crypts by their larger size and elliptical shape. The incidence, distribution and morphology of colon tumors were recorded. The majority of ACF were present in the middle and distal colon of DMH-treated rats and their number increased with time. By the 4th week, 91.5\% ACF were composed of one or two crypts and $8.5 \%$ had three or more crypts, while by the 30 th week $46.9 \%$ ACF had three or more crypts. Thus, a progression of ACF consisting of multiple crypts was observed from the 4 th to the 30th week. Nine well-differentiated adenocarcinomas were found in 10 rats by the 30th week. Seven tumors were located in the distal colon and two in the middle colon. No tumor was found in the proximal colon. The present data indicate that induction of ACF by DMH in the short-term ( 4 weeks) assay was correlated with development of well-differentiated adenocarcinomas in the medium-term (30 weeks) assay.

\section{Introduction}

Colorectal cancer is an increasingly important cause of cancer death in Brazil $(1,2)$. To establish better methods for the clinical

\section{Key words}

- Aberrant crypt foci

- Preneoplastic lesion

- Colon carcinogenesis 
discrete microscopic mucosal lesions, like aberrant crypt foci (ACF), to malignant tumors $(3,4)$.

ACF are focal lesions of the colonic mucosa composed of one to several enlarged crypts, which are specifically induced by colon carcinogens $(5,6)$. Easily identified in methylene blue-stained whole-mount preparations under a dissecting microscope, they are usually quantified as number per animal or per colon and used as early indicators of colon carcinogenesis $(3,7,8)$. However, no correlation between the number of ACF and the incidence of adenocarcinomas was found in some studies $(9,10)$. Evaluation of the predictive value of such precursor lesions as surrogates for neoplasia is increasingly needed in the context of carcinogen or anticancer drug evaluation $(11,12)$.

The genotoxic chemical dimethylhydrazine (DMH) is one of the agents most frequently used in experimental models of colon carcinogenesis in rodents (13-15). It is a complete carcinogen that induces the initiation and promotion steps of carcinogenesis yielding macroscopically visible neoplasms in a dose-dependent manner $(13,15)$. Thus, the DMH-induced colon cancer model in rats is a good tool to investigate the relationship of ACF to colon cancer. To clarify whether ACF are directly related to colon carcinogenesis, this study was carried out to evaluate the relationship between a shortterm (4 weeks) and medium-term ( 30 weeks) assay in a model of colon carcinogenesis induced by $\mathrm{DMH}$ in the rat.

\section{Material and Methods}

\section{Animals and chemicals}

Male Wistar rats aged 6 weeks and weighing about $160 \mathrm{~g}$ were obtained from the Paraná Institute of Technology (TECPAR, Curitiba, PR, Brazil). The animals were housed five per polypropylene plastic cage covered with metal grids in a temperature- controlled room $\left(21-25^{\circ} \mathrm{C}\right)$ with a 12-h light/ 12 -h dark cycle. They were maintained on a Nuvilab-CR1 basic diet (Nuvital, Curitiba, PR, Brazil) and tap water ad libitum. 1,2Dimethylhydrazine was purchased from Sigma (St. Louis, MO, USA). DMH (40 mg/ kg body weight) was dissolved immediately in PBS before use in $1 \mathrm{mM}$ EDTA and the $\mathrm{pH}$ of the solution brought to $\mathrm{pH} 6.5$.

\section{Experimental design}

After 10 days of acclimation the rats were randomly assigned to four groups of 10 animals each. Two groups received four subcutaneous injections of DMH $(40 \mathrm{mg} / \mathrm{kg}$ body weight, twice a week for 2 weeks). Control groups received injections of PBS plus the vehicle (EDTA). One DMH-treated group and one control group were killed at the end of the 4th week (short-term assay). The other DMH-treated group and its respective control were maintained without any further treatment until the 30th week (medium-term assay). All groups were sacrificed by diethyl ether inhalation. This protocol was approved by the Ethics Committee on Care and Use of Laboratory Animals of the National Research Council.

\section{Necropsy and tissue preparation for aberrant crypt foci counting}

At autopsy the entire colon was removed, opened longitudinally and rinsed in saline. Cecum was excised and the remaining colon was divided into three segments of $8 \mathrm{~cm}$ each, referred to as proximal colon (adjacent to the cecum), middle colon and distal colon (adjacent to the rectum). Each segment was fixed flat on filter paper in $10 \%$ buffered formalin for $24 \mathrm{~h}$ and then stained with $0.1 \%$ methylene blue in PBS for $20 \mathrm{~min}$ for ACF counting. Specimens were carefully examined, mucosal side uppermost, by two pathologists at $40 \mathrm{X}$ magnification under a compound light microscope. The number of ACF 
was determined, and their distribution pattern along the colon and crypt multiplicity were scored. Samples for histological analysis were collected and histological sections were cut perpendicular to the muscularis mucosa and stained with hematoxylin and eosin. The material was analyzed for the presence and degree of dysplasia according to Siu et al. (16).

\section{Tumor analysis}

The presence of tumors was carefully noted in terms of number, size and location along the colon. Samples for histological study were processed, sectioned at 4-5 $\mu \mathrm{m}$ and stained with hematoxylin and eosin. The neoplasms were classified according to the histopathological classification proposed by Sunter et al. (13).

\section{Statistical analysis}

Comparisons of ACF number and crypt multiplicity between the short-term (4 weeks) and medium-term (30 weeks) assays were evaluated for significance by the Fisher exact method. The differences were considered significant when $\mathrm{P}<0.05$.

\section{Results and Discussion}

ACF were stereoscopically distinguished from normal crypts by their darker staining and larger size, elliptical shape, thicker epithelial lining, and larger pericryptal zone (Figure 1A). No ACF were detected in control rats. They were observed only in DMHtreated rats.

The distribution and temporal evolution of ACF in the colon are presented in Figure 2. The majority of ACF were present in the middle and distal colon both in the shortterm (4 weeks) and medium-term ( 30 weeks) assays, demonstrating that ACF are not random lesions of the rodent colon treated with genotoxic colon carcinogens (12).

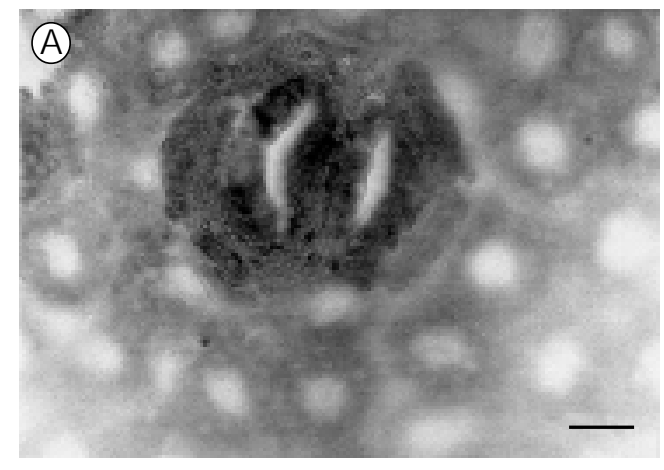

Figure 1. Dimethylhydrazine induced aberrant crypt foci (ACF) in rats. A, Methylene bluestained ACF consisting of two large, elliptical crypts in the short-term (4 weeks) assay. B, Histological pattern of ACF showing dysplasia in the medium-term (30 weeks) assay (H\&E). Bar $=25 \mu \mathrm{m}$.
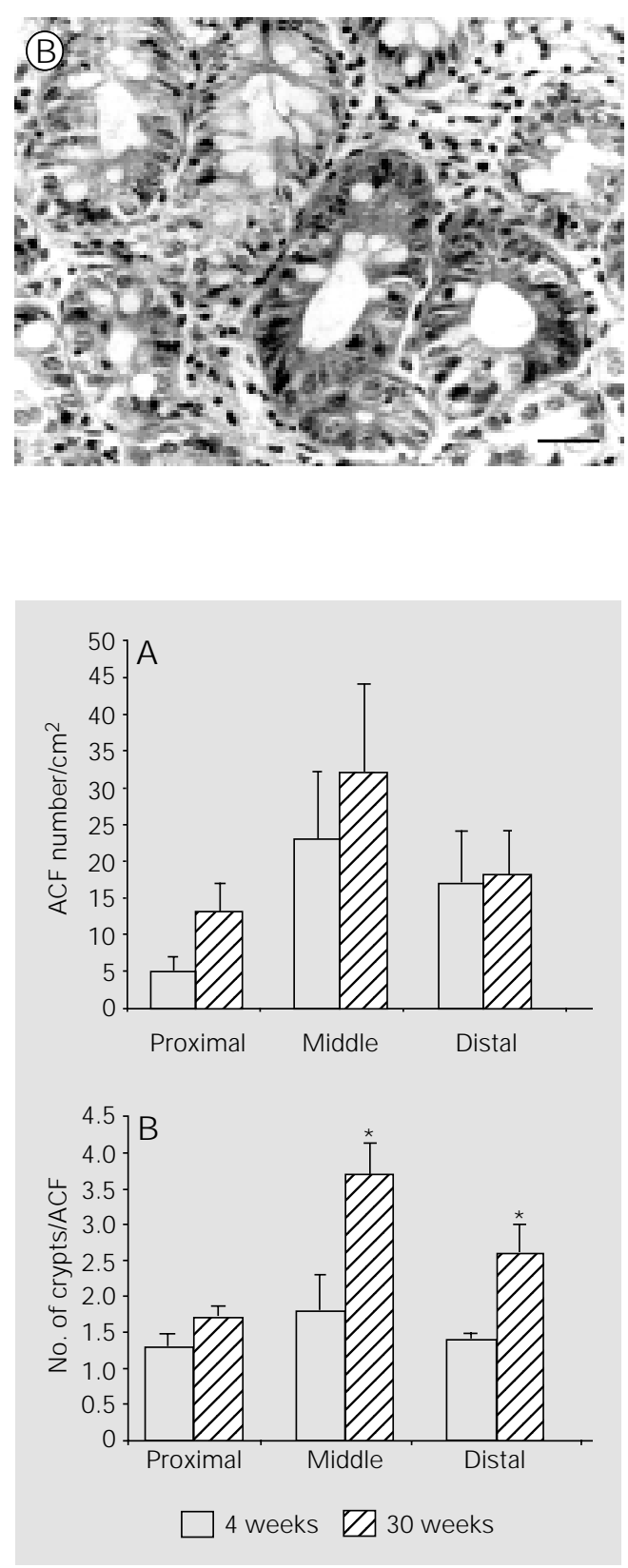

Figure 2. Aberrant crypt foci (ACF) number $(A)$ and number of crypts per ACF (B) in the proximal, middle and distal colon in the short-term (4 weeks) and medium-term (30 weeks) assays. $* \mathrm{P}<0.01$ compared to 4 weeks (Fisher test). 
No significant differences in the number of $\mathrm{ACF} / \mathrm{cm}^{2}$ were observed between the shortterm (4 weeks) and medium-term (30 weeks) assays, especially in the middle and distal colon (Figure 2A). These findings support the view that ACF represent focal morphological alterations induced by DMH in the colonic mucosa and this may reflect the initiation step of carcinogenesis $(3,8)$.

The number of crypts per ACF increased significantly from the 4 th to the 30 th week in the middle and distal colon (Figure 2B). At the 4th week $91.5 \%$ ACF had one or two crypts and only $8.5 \%$ ACF had three or more crypts, while at the 30 th week $46.9 \%$ ACF had three or more crypts (Table 1). This progression of ACF morphology consisting of multiple crypts observed from the 4th to the 30th week may correspond to the promotion step of colon carcinogenesis $(17,18)$.

Histologically, ACF also presented progressive changes. At the 4th week they exhibited hyperplastic features, while mild to

Table 1. Number of aberrant crypt foci (ACF) and crypt multiplicity (\%) in the short-term (4 weeks) and medium-term (30 weeks) assays from rats treated with DMH.

\begin{tabular}{lcccc}
\hline \multirow{2}{*}{ Groups } & Total No. of ACF & \multicolumn{3}{c}{ No. of foci containing } \\
\cline { 3 - 5 } & & 1 crypt & 2 crypts & $>3$ crypts \\
\hline $\begin{array}{l}\text { DMH - 4 weeks } \\
(\mathrm{N}=10)\end{array}$ & $333.2 \pm 94.8$ & $\begin{array}{c}199.5 \pm 59.8 \\
(59.9)\end{array}$ & $\begin{array}{c}105.3 \pm 29.6 \\
(31.6)\end{array}$ & $\begin{array}{c}28.4 \pm 8.5 \\
(8.6)\end{array}$ \\
$\begin{array}{l}\text { DMH - 30 weeks } \\
(\mathrm{N}=10)\end{array}$ & $599.3 \pm 180.1$ & $\begin{array}{c}176.3 \pm 43.0 \\
(29.4)\end{array}$ & $\begin{array}{c}142.2 \pm 35.8 \\
(23.7)\end{array}$ & $\begin{array}{c}280.4 \pm 83.1 * \\
(46.9)\end{array}$ \\
\hline
\end{tabular}

$\mathrm{DMH}$ - dimethylhydrazine. Values are reported as means $\pm \mathrm{SD}$. $* \mathrm{P}<0.05$ compared to DMH - 4 weeks (Student t-test). severe dysplasia was observed at the 30th week (Figure 1B).

Nine tumors were found in 8 of $10 \mathrm{DMH}-$ treated rats examined at the 30 th week. Seven tumors were located in the distal colon and two in the middle colon, which were the sites most populated by ACF. Thus, ACF and colon cancer developed at the same sites in the colonic mucosa of DMH-treated rats, meaning that they may be sequentially related biological events $(3,7,8)$. Interestingly, all tumors were group I well-differentiated adenocarcinomas according to Sunter's classification (13). These findings indicate that ACF may be regarded as early morphological markers in the pathway for well-differentiated tumors in colon carcinogenesis (14). In contrast to other studies (13-15), we did not find tumors in the proximal colon and no tumor was of the poorly differentiated, mucin-secreting histological type. These results may be related to the lower dose of DMH used in the present study compared to previous reports (13-15).

Overall, the results of the present study demonstrate that induction of ACF by DMH in the short-term (4 weeks) assay was correlated with development of well-differentiated adenocarcinomas in the medium-term (30 weeks) assay. The observed relationship supports the view that ACF are precursor lesions for colon tumors and can be used in short-term assays as surrogate end-points to assess carcinogenic or anticancer activity in chemically induced models of colon carcinogenesis.

\section{References}

1. Ministério da Saúde (1999). Estimativa da Incidência e Mortalidade por Câncer no Brasil. INCa, Rio de J aneiro, RJ , Brazil.

2. Medrado-Faria MA, Almeida JWR \& Zanetta DMT (2001). Gastric and colorectal cancer mortality in an urban and industrialized area of Brazil. Revista do Hospital das Clínicas, 56: 47-52.
3. Bird RP (1995). Role of aberrant crypt foci in understanding the pathogenesis of colon cancer. Cancer Letters, 93: 55-71.

4. Takayama T, Katsuki S, Takahashi Y, Ohi M, Nojiri S, Sakamaki S, Kato J , Kogawa K, Miyake H \& Niitsu Y (1998). Aberrant crypt foci of the colon as precursors of adenoma and cancer. New England J our- nal of Medicine, 339: 1277-1284.

5. Bird RP (1987). Observation and quantification of aberrant crypts in the murine colon treated with a colon carcinogen: Preliminary findings. Cancer Letters, 37: 147-151.

6. McLellan KR \& Bird RP (1988). Aberrant crypts: Potential preneoplastic lesions in 
the murine colon. Cancer Research, 48: 6187-6192.

7. McLellan EA, Medline A \& Bird RP (1991). Sequential analyses of the growth and morphological characteristics of aberrant crypt foci: putative preneoplastic lesions. Cancer Research, 51: 5270-5274.

8. Fenoglio-Preiser $\mathrm{CM} \&$ Noffsinger $A$ (1999). Aberrant crypt foci: a review. Toxicologic Pathology, 27: 632-642.

9. Hardman WE, Cameron IL, Heitman DW \& Contreras E (1991). Demonstration of the need for end point validation of putative biomarkers: Failure of aberrant crypt foci to predict colon cancer incidence. Cancer Research, 51: 6388-6391.

10. Carter J W, Lancaster HK, Hardman WE \& Cameron IL (1994). Distribution of intestine-associated lymphoid tissue, aberrant crypt foci and tumors in the large bowel of 1,2-dimethylhydrazine-treated mice. Cancer Research, 54: 4304-4307.

11. Wargovich MJ \& Purewal M (1995). Bio- markers of gastrointestinal neoplasia. In: Rustgi AK (Editor), Gastrointestinal Cancers: Biology, Diagnosis and Therapy. Lippincott-Raven, Philadelphia, PA, USA.

12. Williams GM (1999). Chemically induced preneoplastic lesions in rodents as indicators of carcinogenic activity. In: McGregor DB, Rice J M \& Venitt S (Editors), The Use of Short- and M edium-Term Tests for Carcinogens and Data on Genetic Effects in Carcinogenic Hazard Evaluation. IARC Scientific Publications, Lyon, France.

13. Sunter JP, Appleton DR, Wright NA \& Watson AJ (1978). Pathological features of the colonic tumors induced in rats by the administration of 1,2-dimethylhydrazine. Virchows Archiv. B, Cell Pathology, 29: 211-223.

14. Park HS, Goodlad RA \& Wright NA (1997). The incidence of aberrant crypt foci and colonic carcinoma in dimethylhydrazinetreated rats varies in a site-specific manner and depends on tumor histology. Can- cer Research, 57: 4507-4510.

15. Sequeira J L, Kobayasi SK \& Rodrigues MAM (2000). Early and late effects of wound healing on development of colon tumors in a model of colon carcinogenesis by 1,2 dimethylhydrazine in the rat. Pathology, 32: 250-252.

16. Siu I-M, Pretlow TG, Amini SB \& Pretlow TP (1997). Identification of dysplasia in human colonic aberrant crypt foci. American J ournal of Pathology, 150: 1805-1813.

17. Zhang X-M, Stamp D, Minkin S, Medline A, Corpet DE, Bruce WR \& Archer MC (1992). Promotion of aberrant crypt foci and cancer in rat colon by thermolyzed protein. J ournal of the National Cancer Institute, 84: 1026-1030.

18. Magnuson BA, Carr I \& Bird RP (1993). Ability of aberrant crypt foci characteristics to predict colonic tumor incidence in rats fed cholic acid. Cancer Research, 53: 4499-4504. 